\title{
Der Herausgeber
}

14 Nummern hat Prof. Dr. Horst Prießnitz herausgegeben und den Newsletter zu dem gemacht, was er ist: ein reichhaltiges, informatives Organ der Gesellschaft für Australienstudien, das auch einen Einblick in deren Leben gibt. Welche Veranstaltungen fanden statt, wo liegen die Interessen der Mitglieder, aus welchen Bereichen kommen sie? Mein Vorgänger hat dankenswerterweise für eine Kontinuität gesorgt: der Newsletter wird weiterhin an der Bergischen Universität GHS Wuppertal gedruckt und von dort versandt. Dafür sind wir ihm und der Universität zu Dank verpflichtet.

Der Newsletter wird weiterhin das Leben der GASt wiederspiegeln. Aber jeder Herausgeber wird versuchen, ihn noch ansprechender und informativer zu machen. Damit ist keine Akademisierung gemeint, auch wenn die Mehrzahl der Mitglieder aus den Hochschulen kommt. Aber viele kommen aus den Medien, den zoologischen Gärten, dem Museumsbereich, dem Kulturmanagement, der Wirtschaft oder internationalen Institutionen. Was kann der Herausgeber machen, um eine so vielfältige Leserschaft anzusprechen, zumal der Newsletter nur einmal pro Jahr erscheint?

Ich denke an dieses: Erstens werde ich für jedes Jahr einen Themenschwerpunkt setzen, der aus unterschiedlichen Gründen aktuell ist und aus vielen Perspektiven beleuchtet werden kann. Zweitens soll der Newsletter der Vielfalt der Mitglieder und Leser Raum geben, sich darin wieder zu erkennen und interessante Beiträge zu liefern. Da der Schwerpunkt andere Themen nicht ausschließt, finden sich in der Struktur auch die bekannten Sektionen wieder. Schließlich wird der Newsletter einen aktiveren Rezensionsteil und gelegentlich einen Resourcenteil—so zum Webenthalten, um auf wichtige Publikationen und Informationsquellen aufmerksam zu machen. Ich erwarte ein aktives Echo, wobei Autoren nicht nur aus dem Kreis der Gesellschaft kommen werden. Der Newsletter ist offen für Beiträge von außerhalb und aus Ländern, in denen Australien Gegenstand des öffentlichen und akademischen Interesses ist. Die Klagenfurter Konferenz hat gezeigt, daß wir-neben Australien-über Deutschland, Österreich und der Schweiz hinaus mit Kollegen aus den Ländern Mitteleuropas ebenso kooperieren sollen, wie mit denen aus Westeuropa.

Nun zu diesem Heft. Die Wahl eines Schwerpunktes war einfach, denn die Föderation der britischen Kolonien zum Commonwealth of Australia im Jahre 1901 ist das herausragende Datum. Der Newsletter beleuchtet dieses Ereignis aus der Sicht der Politik, Wirtschaft, Politik, Geschichte, Literatur und Sprache. Als Vorgabe für die Autoren wurde lediglich angeregt, auch auf Fragestellungen für das 3. Jahrtausend einzugehen oder darauf hinzuweisen.

\section{Last but not least der Schwerpunkt für Nummer 16, 2002 (Redaktionstermin 1. Juni 2002): "MOSAIK, MELTING POT UND SYNKRETISMUS. AUSTRALISCHER MULTIKULTURALISMUS IM 3. JAHRTAUSEND."}

Der Zusammenhang verschiedenet ethnischen oder anders definierter Gruppen eines multikulturellen Australien verdient eine Neubetrachtung. Zuviel ist seit den frühen 90er Jahren passiert. Ist Multikulturalismus noch das unbestrittene Gesellschaftsbild? Welche Alternativkonzepte haben sich herauskristallisiert? Wie spiegeln sich damit verbundene Themen in der Literatur, in Film und Fernsehen, in der Kunst wider? Welchen Status hat die ethnische Literatur? Wie reagiert die Sprachpolitik auf nichtenglischer Sprachen heute? Wie gehen Industrie, Handel und Politik mit Pluralisimus um? Inwieweit lenken staatliche Fördereinrichtungen (z.B. Arts Council, ATSIC, etc.) die Entwicklungen? Diese Fragen seien hier zur Anregung vorgegeben. 